\title{
Oxford-AstraZeneca COVID-19 vaccination induced lymphadenopathy on [18F]Choline PET/CT—not only an FDG finding
}

\author{
Ayah Adel Nawwar ${ }^{1,2}$ (D) Julie Searle ${ }^{1,3} \cdot$ Rajiv Singh ${ }^{1,3} \cdot$ lain Douglas Lyburn 1,3,4 \\ Received: 16 February 2021 / Accepted: 22 February 2021 / Published online: 4 March 2021 \\ (C) The Author(s), under exclusive licence to Springer-Verlag GmbH, DE part of Springer Nature 2021
}

Large-scale worldwide COVID-19 vaccination programs are being rapidly deployed. Many patients with malignancy, a high-risk group, have recently had the first dose of the vaccine. A 75-year-old male with prostate cancer and biochemical recurrence underwent [18F]Choline PET/CT for disease assessment. No choline avid malignancy was identified; however, avid left axillary nodes were noted.
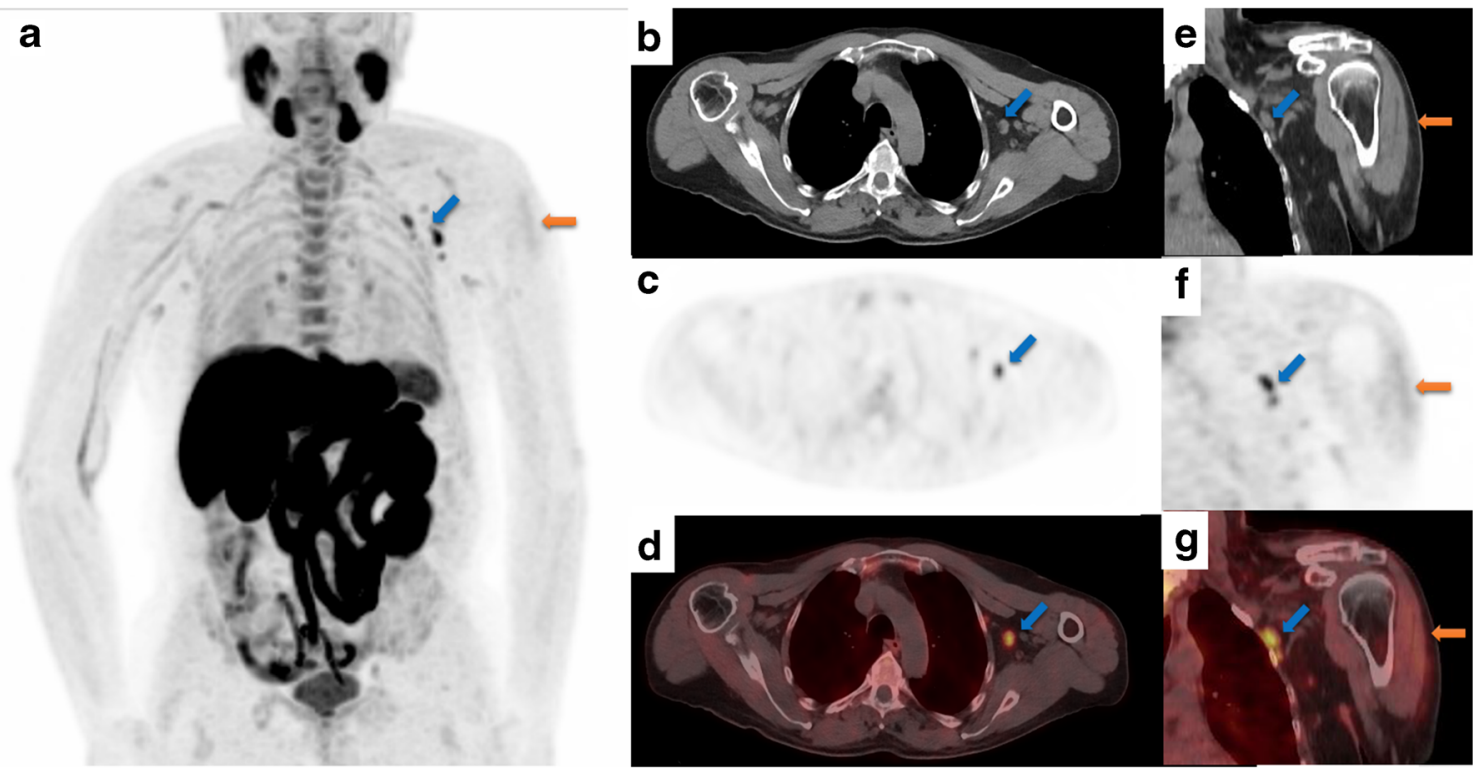

[18F]Choline PET/CT, maximal intensity projection (MIP)-A, demonstrates mild ill-defined uptake in the left arm (orange arrow) and areas of intense focal uptake in the left axilla (blue arrow). Tracer injection was via the right

This article is part of the Topical Collection on Image of the month

Ayah Adel Nawwar

ayah.nawwwar@doctors.net.uk

Cobalt Medical Charity, Linton House, Thirlestaine Rd, Cheltenham GL53 7AS, UK (contralateral) antecubital fossa, hence not a potential cause. The mildly avid symmetrical small volume mediastinal and hilar nodes are a common benign finding [1]. Axial CT, $[18 \mathrm{~F}]$ Choline PET, and fused [18F]Choline PET/CT of the

2 Clinical Oncology and Nuclear Medicine department, Cairo University, Cairo, Egypt

3 Gloucestershire Hospitals NHS Foundation Trust, Gloucestershire, UK

4 Cranfield University, Bedford, UK 
chest (B, C, D) demonstrate uptake within level I/II left axillary nodes. Coronal CT, [18F]Choline PET, and fused $[18 \mathrm{~F}]$ Choline PET/CT of the left upper arm demonstrate uptake within the deltoid muscle $(\mathbf{E}, \mathbf{F}, \mathbf{G})$.

The patient gave a history of receiving the OxfordAstraZeneca covid-19 vaccination in the left upper arm 3 days earlier. An uptake in the left upper arm (deltoid) is in keeping with this. Clinical correlation supports the nodal uptake to be reactive, related to the recent vaccination. Similar findings were recently described with Pfizer-BioNTech COVID-19 vaccination but on [18F]FDG-PET/CT [2].

This case introduces a new PET/CT pitfall associated with the current COVID-19 pandemic vaccination programs on $[18 \mathrm{~F}]$ Choline, which if not made aware of, may result in incorrect image interpretation and inadvertent upstaging of disease.

\section{Declarations}

Ethics approval and informed consent All procedures performed involving the human participant were in accordance with the ethical standards of the institutional and/or national research committee and with the 1964 Helsinki declaration and its later amendments or comparable ethical standards. Informed consent was obtained from the patient.

Competing interests The authors declare no competing interests.

\section{References}

1. Calabria F, Chiaravalloti A, Schillaci O. 18F-choline PET/CT pitfalls in image interpretation: an update on 300 examined patients with prostate cancer. Clin Nucl Med. 2014;39:122-30.

2. Eifer M, Eshet Y. Imaging of COVID-19 vaccination at FDG PET/ CT. Radiology. 2021;210030.

Publisher's note Springer Nature remains neutral with regard to jurisdictional claims in published maps and institutional affiliations. 